\title{
The Effect of Kick Type on the Relationship between Kicking Leg Muscle Activation and Ball Velocity
}

\author{
Ali Onur Cerrah ${ }^{1}$, Abdullah Ruhi Soylu², Hayri Ertan ${ }^{1}$, Adrian Lees ${ }^{3}$
}

\begin{abstract}
Affiliations: 'Anadolu University, Faculty of Sport Sciences, Department of Coaching Education, Eskisehir, Turkey, ${ }^{2}$ Hacettepe University, School of Medicine, Biophysics Department, Ankara, Turkey, ${ }^{3}$ Emeritus Professor
\end{abstract}

Correspondence: A.O. Cerrah, Anadolu University, Faculty of Sport Sciences, Iki Eylül Kampüsü, Spor Bilimleri Fakültesi, Tepebaşı, Eskişehir, Turkey. E-mail: aocerrah@anadolu.edu.tr

ABSTRACT This study aimed to identify the effects of different kick types on the relationship between kicking leg muscle activation and ball velocity. The muscle activation of selected knee extensor and flexor muscles of 10 amateur soccer players were measured using electromyography during the performance of six maximal soccer kick types. The highest ball velocity was achieved by the instep kick ( $\left.96.2 \mathrm{~km} / \mathrm{hr}^{-1}\right)$, followed by the lofted kick, the inside curve kick, the outside kick, the outside curve kick, and finally the inside kick (81.3 $\left.\mathrm{km} / \mathrm{hr}^{-1}\right)$. There were significant positive correlations between muscle activation and ball velocity for the vastus lateralis and lofted (0.765), inside curve (0.792) and instep kicks (0.788), and for the gastrocnemious with the outside kick (0.796). Non-significant correlations between muscle activation and ball velocity exhibited a trend such that they were positive for the vastus medialis and vastus lateralis but negative for the biceps femoris and gastrocnemious for inside-foot-dominated kicks, while this trend was reversed for outside-foot-dominated kicks. According to results, the noted trends can be explained by the change in muscle activation patterns required to orientate the foot for each type of kick; this has implications for players' training activities.

KEY WORDS soccer, kick type, electromyography, ball velocity

$@$ MJSSMontenegro

MUSCLE ACTIVATION AND BALL VELOCITY RELATIONSHIP

http://mjssm.me/?sekcija=article\&artid=150

\section{Introduction}

Various kick types are used in soccer to kick a stationary ball and can be classified as: (1) inside, (2) lofted, (3) inside curve, (4) instep, (5) outside, and (6) outside curve kicks (Bauer, 1993). All these kick types can be used for scoring and players who are proficient in the use of each increase their possibility of scoring a goal because it reduces the time the goalkeeper has to react (Sterzing et al., 2009).

To our knowledge, the ball velocities of only three kick types (inside, instep, outside) have been analysed in previous studies (Nunome et al. 2002; Asai et al. 2002; Katis \& Kellis, 2010). The velocity of the ball has been used as a key indicator for total kick performance (Kellis \& Katis, 2007; Lees \& Nolan, 1998). Katis and Kellis (2010) determined ball velocity in youth players and found the inside kick velocity $\left(70.6 \pm 5.8 \mathrm{~km} / \mathrm{h}^{-1}\right)$ to be higher than that of the outside kick $\left(65.2 \pm 5.4 \mathrm{~km} / \mathrm{h}^{-1}\right)$. Other studies (Kawamoto et. al., 2007; Levanon \& Dapena, 1998; Nunome et al., 2002) also showed that the instep kick generates higher ball speeds than the inside and outside kick types do. However, no study has reported ball velocities from the other types of kick that are defined in the current study.

As the kick type changes from the inside kick to the outside curve kick, movement patterns and limb positions change. For example, when kicking the ball using the inside kick, the player needs to outwardly rotate and extend the hip joint, flex the knee and evert and plantar flex the ankle. In contrast, kicking the ball using the outside curve type requires the player to inwardly rotate and extend the hip joint, to flex the knee, and to invert and plantar flex the ankle. 
Movement patterns and the position of the limbs for different kick types occur as a result of muscular contractions surrounding the hip, knee, and ankle joints. Several studies have been conducted to explain the muscle activation patterns that occur during kicking by electromyography (EMG) but only for the inside and instep kicks (Brophy et. al., 2007; Cerrah et al., 2011; Dorge et al. 1999; Scurr et. al., 2011). The soccer kick is considered to be the result of the simultaneous activity of many muscles connecting segments and causing joint movement. Some of these muscles act as antagonists during the kick and are thought to limit performance. This is termed the "soccer paradox" (Clarys et. al., 1984; Bollens et al., 1987; De Proft et al., 1988; McCrudden \& Reilly, 1993).

In the kicks performed with the inside and outside parts of the foot, the lower limb extremity joints show higher rotations in the transverse and frontal planes, which are necessary in order to bring the foot to the appropriate position (inverted, dorsi-flexed, and supinated) for ball contact, in comparison with the instep kick (Kellis and Katis, 2010; Nunome et al., 2002). This means that, while the sagittal plane movements of the lower limb show similarity between different types of kick, the frontal and transverse joint rotations through the forward motion of the kicking leg differ between the differing types of kick. Consequently, muscle activation might also change according to kick type. To date, there have been no studies that have investigated the relationship between the level of muscle activation and ball velocity for different kick types.

Our purpose, therefore, is to define the relationship between the muscle activation of selected muscles operating around the kicking leg knee with the ball velocities produced during the maximal performance of different kick types. We hypothesized that a relationship between muscle activation and ball velocity will exist and will be affected by kick type.

\section{Methods}

Ten amateur male soccer players (height: $177.4 \pm 6.6 \mathrm{~cm}$, mass: $74.7 \pm 6.4 \mathrm{~kg}$, age: $24.3 \pm 2.7$ years) who could successfully perform the kick types investigated volunteered to participate in the current study. All participants were soccer players of the same team competing in the local amateur league, and their training frequency was three times a week with a competition on weekends. Their descriptive statistics are summarized in Table 1. None of the participants reported any previous injury to their lower limbs. All players were informed about the protocol of the training, possible benefits, and risks, and gave their written informed consent. This study was approved by the University Ethics Committee (Protocol Number: PR-08-12-04-08) and carried out in accordance with the Declaration of Helsinki.

\begin{tabular}{cccccccc} 
TABLE 1 Descriptive statistics for amateur soccer players $(\mathrm{n}=10)$ \\
Age (years) & $\begin{array}{c}\text { Athletic } \\
\text { History } \\
\text { (years) }\end{array}$ & \multicolumn{9}{c}{ Position } & & & Kicking Leg \\
\hline mean \pm SD & mean \pm SD & GK & D & MF & F & right & left \\
$24.3 \pm 2.7$ & $12.9 \pm 3.0$ & 1 & 2 & 2 & 5 & 10 & 0 \\
\hline
\end{tabular}

Note: GK - goal keeper, D - defence, MF - midfielder, F - forward

The soccer kick test was performed following a warm up (10 min jog) and five soccer-specific stretching exercises $(10 \mathrm{~min})$ in an indoor sport area. For standardization purposes, players were asked to perform the inside kick ( $0-10^{\circ}$ approach angle), lofted kick ( $0-10^{\circ}$ approach angle), inside curve kick (30-45 approach angle), in-step kick ( $0-10^{\circ}$ approach angle), outside kick ( $0-10^{\circ}$ approach angle) and outside curve kicks (30$45^{\circ}$ approach angle) in a random order. The descriptions of kick types are given in Table 2 . The inside kick was performed with one step and the remaining kicks were performed with two advancing steps towards a stationary ball aimed at a target (width $3.00 \mathrm{~m}$, height $2.44 \mathrm{~m}$ ) $15 \mathrm{~m}$ away with a full size (number 5) soccer ball approved by the International Football Federation (Masuda et al., 2003). Ball pressures were adjusted to 11 psi with a pressure measurement device (Rucanor, Netherland). Participants were asked to kick the ball with maximal velocity and accuracy so as to strike a target. A total of three kicks were analysed, and the data averaged. Ball velocity was measured with a radar gun (Bushnell Velocity Speed Gun, USA) held behind the goal. All kicks were recorded with a video camera (Canon $\mathrm{Hg} 21$ ) at $50 \mathrm{~Hz}$ and the toe-off to impact time during kick identified with video analyses software for sport (Kinovea 0.8.15). A mechanical switch was attached to the shoe of the players to accurately measure the moment of the contact between the player's foot and ball. The signal obtained from the contact of the foot was superimposed on the EMG recordings.

The EMG measurement sites were prepared according to SENIAM recommendations (Hermens et al., 2000). The centre-to-centre distances between two Circular $\mathrm{Ag} / \mathrm{AgCl}$ surface electrodes (Blue Sensor Electrodes, Noraxon) was $2 \mathrm{~cm}$ and were placed longitudinally with respect to the underlying muscle fibre arrangement. The muscles investigated were the $m$. rectus femoris (RF), the $m$. vastus lateralis (VL), the $m$. vastus medialis (VM), the long head of $m$. biceps femoris (BF), and the m. medial gastrocnemius (GAS). Furthermore, the reference electrode was placed on the lateral malleolus of the fibula. Only five muscles' EMG recording were analysed because these muscles were mostly analysed in previous studies and were more suitable for dynamic action such as soccer kicks. Moreover, the maximum isometric voluntary contractions (MVIC) of the quadriceps (RF, VL, VM) was assessed by knee extension when sitting with the knee at a $65^{\circ}$ angle $\left(0^{\circ}\right.$ 
TABLE 2 Description of kick types

Kick Types

Description

Inside Kick

The kicking leg is rotated outward from the hip joint, and the ankle joint is rotated outward to form approximately a $90^{\circ}$ angle of the foot to the direction of the kick. The contact area is between the metatarsals and calcaneus on the medial aspect of the foot and close to the centre of mass for the ball

Lofted Kick

The kicking leg is rotated outward from the hip joint, and the ankle joint is rotated outward to form approximately a $45^{\circ}$ angle of the foot to the direction of the kick with the toe close to the ground. The contact area for the foot is around the metatarsals, and for the ball, underneath its centre of mass

Inside Curve Kick

The kicking leg is rotated outward from the hip joint and the ankle joint is rotated outward to form approximately a $45^{\circ}$ angle of the foot to the direction of the kick. To spin the ball with the right foot, the contact area is around the metatarsals for the foot and underneath, but to the outside of the centre of mass for the ball

Instep Kick

The kicking leg is rotated slightly outward from the hip joint, and the ankle joint is plantar flexed so the toes point to the ground. The contact area for the foot is around the laces of the soccer shoe and for the ball, close to its centre of mass

The kicking leg is oriented inward from the hip joint, and the ankle joint is rotated outward to form approximately a $30-45^{\circ}$ angle of
the foot to the direction of the kick with the toe close to the ground. The contact area for the foot is slightly above the metatarsals, and for the ball, underneath but to the outside of its centre of mass

The kicking leg is oriented inward from the hip joint, and the ankle joint is rotated outward to form approximately a $30-45^{\circ}$ angle of

Outside Curve Kick the foot to the direction of the kick. To spin the ball with the right foot, the contact area for the foot is slightly above the metatarsals and for the ball, underneath but to the inside its centre of mass.

full extension) against a resistance, while the MVIC of hamstrings (long head of BF) was assessed in a prone position with the knee at a $30^{\circ}$ angle against a resistance (Kellis and Baltzopoulus, 1988). For the MVIC of the GAS, participants were asked to perform plantar flexion against a resistance when in the supine position (Cerrah et al., 2011). A Biovision EMG system was used to record EMG signals (Biovision, Germany) with maximum intra-electrode impedance and minimum CMMR of $6 \mathrm{~K}-\mathrm{Ohm}$ and $95 \mathrm{~dB}$, respectively. Analogue signals were pass band filtered between $20-500 \mathrm{~Hz}$ and digitized by a 12-bit A/D converter at $1000 \mathrm{~Hz}$. To display the activation patterns of the muscles, which occur in six different kick types, the filtered EMG signals were smoothed using a $40 \mathrm{~ms}$ root-mean-square filter. They were also normalized by the maximal EMG activity recorded during MVICs for each participant (Cerrah et al. 2011). The mean of the normalized EMG data from the beginning of toe-off of the kicking leg to ball contact was averaged over the three kicks and repeated for each of the six different kick types to give a value for muscle activation (Table 4) and subsequently correlated with ball velocity.

Descriptive statistics were applied to identify the characteristics of the participants and groups. Mean scores were calculated for each participant's three kicks for each type of kick and then averaged across each group. All data were normally distributed (Shapiro Wilk). Ball velocities are expressed as mean \pm SD. Two oneway analyses of variance (ANOVA) were performed to firstly analyse differences between ball velocities and kick types, and secondly to analyse differences between muscle activation and kick types. The level of significance was set at $\mathrm{p}<0.05$. When a significant interaction was detected, data were subsequently analysed using a Tukey post-hoc test. Pearson Correlation was used to correlate muscle activation and ball velocity for each muscle and the six kick types. Pearson's Correlation coefficient was interpreted according to Domholdt (2000): $0.00-0.25=$ little if any correlation, $0.26-0.49=$ weak correlation, $0.50-0.69=$ moderate correlation, $0.70-0.89=$ strong correlation, $0.90-1.00=$ very strong correlation.

\section{Results}

The highest ball velocity was achieved for the instep kick followed by the lofted kick, the inside curve kick, the outside kick, the outside curve kick, and finally the inside kick (Table 3). Significant differences occurred between the inside kick and the lofted, inside curve, and instep kicks. Other significant differences occurred for the outside curve kick with the lofted and instep kicks.

\begin{tabular}{ccc}
\hline TABLE 3 Ball velocities from different kick types & \\
& Kick Type & mean \pm SD \\
\hline & Inside & $81.3 \pm 3.9$ \\
& Lofted & $92.3 \pm 8.1 \boldsymbol{\Psi}^{* *}, \mathbf{a}^{*}$ \\
Ball Velocities $\left(\mathrm{km} \cdot \mathrm{h}^{-1}\right)$ & $91.2 \pm 8.0 \boldsymbol{\Psi}^{*}$ \\
& Inside Curve & $96.2 \pm 7.3 \boldsymbol{\Psi}^{* *} \mathbf{a}^{* *}$ \\
& In-step & $87.5 \pm 5.4$ \\
& Outside & $83.5 \pm 5.9$ \\
\hline
\end{tabular}

Note: $\Psi$ - Statistically significant different to the inside kick; $a$ - Statistically significant different to the outside curve kick; ${ }^{*}-\mathrm{p}<0.05 ;{ }^{* *}: \mathrm{p}<0.01$ 
In RF, the highest level of muscle activation occurred for the outside kick and was significantly different from the rest of the kicks (Table 4). In VL, the highest muscle activation occurred for the outside curve kick but was significantly different only from the inside kick. In VM, the highest muscle activation also occurred in the outside curve kick. In BF, the highest muscle activation occurred for the inside kick while in GAS, the highest muscle activation occurred in the lofted kick. The mean muscle activation values for each muscle and the significance of differences with regard to kick types are given in Table 4.

\begin{tabular}{|c|c|c|c|c|c|c|c|}
\hline & Muscles & $\begin{array}{c}\text { Inside } \\
\text { mean } \pm S D\end{array}$ & $\begin{array}{c}\text { Lofted } \\
\text { mean } \pm S D\end{array}$ & $\begin{array}{c}\text { Inside Curve } \\
\text { mean } \pm S D\end{array}$ & $\begin{array}{c}\text { In-step } \\
\text { mean } \pm S D\end{array}$ & $\begin{array}{c}\text { Outside } \\
\text { mean } \pm S D\end{array}$ & $\begin{array}{c}\text { Outside Curve } \\
\text { mean } \pm \text { SD }\end{array}$ \\
\hline \multirow{5}{*}{$\begin{array}{c}\text { Mean } \\
(\%)\end{array}$} & $\mathrm{RF}$ & $0.39 \pm 0.09^{\mathrm{e}^{* * *}}$ & $0.39 \pm 0.06^{\mathrm{e}^{* * *}}$ & $0.36 \pm 0.06^{\mathrm{e} * *}$ & $0.40 \pm 0.07^{\mathrm{e}^{* * *}}$ & $0.57 \pm 0.14$ & $0.36 \pm 0.05^{\mathrm{e}^{* *}}$ \\
\hline & $\mathrm{VL}$ & $0.25 \pm 0.08^{f *}$ & $0.34 \pm 0.11$ & $0.34 \pm 0.09$ & $0.37 \pm 0.09$ & $0.38 \pm 0.12$ & $0.39 \pm 0.12$ \\
\hline & VM & $0.23 \pm 0.09$ & $0.39 \pm 0.10^{\mathrm{a}^{* *}}$ & $0.32 \pm 0.11$ & $0.36 \pm 0.11$ & $0.38 \pm 0.08^{\mathrm{a}^{*}}$ & $0.42 \pm 0.09^{\mathrm{a}^{* *}}$ \\
\hline & $\mathrm{BF}$ & $0.22 \pm 0.08$ & $0.16 \pm 0.06$ & $0.15 \pm 0.05$ & $0.12 \pm 0.07^{\mathrm{a}^{* *}}$ & $0.09 \pm 0.02^{\mathrm{a}^{* *}}$ & $0.10 \pm 0.05^{\mathrm{a}^{* *}}$ \\
\hline & GAS & $0.23 \pm 0.09^{b^{*}}$ & $0.36 \pm 0.12$ & $0.26 \pm 0.09$ & $0.28 \pm 0.09$ & $0.22 \pm 0.04^{b^{* *}}$ & $0.23 \pm 0.04^{b^{*}}$ \\
\hline
\end{tabular}

Note: RF - Rectus Femoris, VM - Vastus Medialis, VL - Vastus Lateralis, BF - Biceps Femoris, GA- Gastrocnemious, MVC - Maximum Voluntary Contraction, a - Statistically significantly different to the inside kick, b - Statistically significantly different to the lofted kick; $\mathbf{c}$ - Statistically significantly different to the inside curve kick; $\mathbf{d}$ - Statistically significantly different to the instep kick; $\mathbf{e}$ - Statistically significantly different to the outside kick; $\mathbf{f}$ - Statistically significantly different to the outside curve kick; ${ }^{*} p<0,05 ;{ }^{* *}: p<0,01$

There were significant positive correlations between muscle activation and ball velocity for VL and lofted, inside curve, and instep kicks, and a significant positive correlation for GAS with the outside kick (Table 5). The inside-dominated kicks (inside, lofted, inside curve, instep) followed a trend of positive correlations (0.560 to 0.792$)$ for VL and VM and negative correlations (-0.057 to -0.601) for BF and GAS. The outsidedominated kicks (outside and outside curve kick), followed an opposite trend of negative correlations for VM $(-0.136$ to -0.292$)$ but positive correlations (0.079 to 0.796$)$ for BF and GAS.

\begin{tabular}{|c|c|c|c|c|c|}
\hline \multirow{2}{*}{ Kick types } & \multicolumn{5}{|c|}{ Muscles } \\
\hline & RF & VM & VL & BF & GAS \\
\hline Inside & -0.038 & 0.629 & 0.311 & -0.513 & -0.601 \\
\hline Lofted & -0.471 & 0.560 & $0.765^{*}$ & -0.057 & -0.058 \\
\hline Inside curve & 0.280 & 0.635 & $0.792^{*}$ & -0.146 & -0.436 \\
\hline In-step & -0.169 & 0.513 & $0.788^{*}$ & 0.313 & -0.070 \\
\hline Outside & -0.463 & -0.136 & 0.361 & 0.0791 & $0.796^{*}$ \\
\hline Outside curve & -0.361 & -0.292 & 0.600 & 0.0845 & 0.673 \\
\hline
\end{tabular}

Note: RF - Rectus Femoris, VM - Vastus Medialis, VL - Vastus Lateralis, BF - Biceps Femoris, GAS - Gastrocnemious, $*: p<0.05$

\section{Discussion}

The aim of this study was to define the relationship between activation characteristics of five muscles around the kicking leg knee and ball velocity for six different kick types. The main findings are that (1) both muscle activation and ball velocity changed as a function of kick type, (2) there was a significant relationship between muscle activation and ball velocity for certain muscles and certain kick types, and (3) there was a trend for the inside-orientated kicks to have muscle activation patterns opposite to those found in the outside orientated kicks.

Different kick types have been found to produce significantly different maximal velocities. The highest velocity $\left(96 \mathrm{~km} / \mathrm{h}^{-1}\right.$, instep) is $18 \%$ greater than the lowest velocity of the inside kick $\left(81 \mathrm{~km} / \mathrm{h}^{-1}\right)$. These data are similar to those reported by Nunome et al., (2006) $\left(94.7 \mathrm{~m} / \mathrm{s}^{-1}\right.$, instep) for experienced players but greater than those reported by Kellis et al. (2006) $\left(88.9 \mathrm{~km} / \mathrm{h}^{-1}\right.$, instep, two-step) for amateur players and lower than those reported by Cometti et al., (2001) (106.4 km/h $\mathrm{h}^{-1}$, free approach) for professional players. Muscle activation also differed significantly between kick types. There is a lack of published data on this variable so it is not possible to make direct comparisons with the literature. However, the differences found suggest that establishing the relationship with ball velocity would be productive.

The relationship between muscle activation and ball velocity has been speculated upon by others (e.g. Kellis and Katis, 2010; Nunome et al., 2002). The different limb motions and postures required to achieve each type of kick would appear to require different muscle activation patterns. In this study, the kick types that required the greatest foot re-orientation (the inside kick and the outside kick) produced the lowest velocities suggesting that the process of re-orientating the foot for ball impact compromises foot (and hence ball) velocity. To our 
knowledge, no study has reported any correlation between muscle activation and ball velocity for a range of commonly used kick types. The correlation between five muscles of the kicking leg and the ball velocities of six different kick types show positive and negative correlations. The significant positive correlations occurred in VM for the fastest of the kicks (instep, inside curve and lofted kicks). This finding implies that knee extensor muscle activation is a significant contributor to kick performance, particularly in those kicks with which the leg is turned outwards to make contact on the inside or medial aspect of the foot. Because of the curved approach, the medial aspect of the foot naturally contacts the ball so it would appear that in these types of kicks the knee extensors are able to operate effectively (i.e. their muscle line of action is not required to deviate in order to produce a medial or lateral re-orientation of the foot for contact). The greater ball velocity may be interpreted in terms of greater muscle activation combined with better functionality of joint action.

A trend was observed between inside (inside, lofted, inside curve, and instep) and outside (outside and outside curve) foot-dominated kicks. The former kicks are observed to produce positive correlations with ball velocity for VM and VL and mainly negative correlations for BF and GAS, while the latter produce an opposite pattern. Those kicks, which are inside-dominated require high levels of knee extensor muscle activation, but reduced activation of the knee flexors as these inhibit knee extension as a characteristic of the "soccer paradox". Outside foot-dominated kicks do not show this feature, which suggests that these types of kicks require fundamentally different movement patterns. They are considered to be the most technically difficult. That they demonstrate lower knee extensor activity combined with greater knee flexor activity is a consequence of the requirement to inwardly rotate the foot for ball contact on the outside of the foot. Reduced knee extensor activity and increased knee flexor activity would negatively impact foot (and hence ball) velocity at impact; therefore, a reduced average ball speed would be expected. This is found for the outside kick, which is an extreme form of this type of kick, but not to the same extent for the outside curve kick. The significant relationship between GAS and ball velocity for the outside kick might be due to the need for greater foot rigidity, leading to better foot-ball interaction. The correlation for the outside curve kick is not significant but follows the same trend suggesting a similar need for enhanced foot rigidity.

Finally, the role of RF is worth noting: it generally has a low negative correlation between muscle activation and ball velocity. This suggests that it is not a prime mover for the technical changes required for the more complex kicks. Because the RF muscle is a two-joint muscle, it could be contributing to the well-executed kick by allowing the upper leg to decelerate. Therefore, elastic energy is transferred from the thigh to the shank. For that reason, the role of RF muscle should be investigated further together with kinematic data in order to identify the contribution this muscle makes during kicking performance.

Activation patterns of muscles for the different kick types used here are similar to those described in other studies, which is that the kick requires a proximal-to-distal segmental movement occurring as a result of motion-dependent moments generated by active involvement of the whole body (Shan and Westerhoff, 2005; Nunome et al., 2002; Lees, 2010).

It has been shown in the current study that even though the general activation patterns are similar, the involvements of the muscles that determine the kicking velocity change according to kick type. This would suggest that some form of training taking into account the findings described above would be beneficial to players.

This study was a preliminary investigation into an under-reported area of soccer performance related to different kick types. Within the limitations of the study, it has been found that VL and VM muscles are significant determinants for the inside, lofted, inside curve and instep kicks while BF and GAS muscles are significant determinants for the outside and outside curve kicks. It seems that the fastest kick types use high activation of knee extensors and low activation for the knee flexors. For the outside kick types, the leg and ankle flexors play a more important role, but to the detriment of the knee extensors.

\section{Conclusion}

The results of this study demonstrate that maximal kicking velocity is dependent on kick type and can in part be explained by the level of muscle activity in leg muscles, which supports the hypothesis of this paper. The muscle activation characteristics of the knee flexors and extensors studied are significant determinants of different kick types. Therefore, a type of muscle training that improves the coordination pattern of muscle activation relevant to the different kick types described could be beneficial to players. Therefore, power training should be suitable for all types of kicks; however, in order to train specific kicking types, resistance band (cable) and coordination training that represent kicking motion could be recommended for players and coaches. Moreover, it could be more beneficial if the resistance trainings are organized regarding the positions of trunk, hip, leg and ankle with active involvement of muscles described in the current study. This study provides baseline findings for further investigation into the changes in muscular activation values and patterns due to kick type, including the influences of age and players' skill level. 


\section{RE F ERENCES}

Asai, T., Carré, M. J., Akatsuka, T., \& Haake, S. J. (2002). The curve kick of a football I: impact with the foot. Sports Engineering, 5(4), 183-192.

Bauer, G. (1993). Soccer Techniques, Tactics and Teamwork: New York Sterling Publishing Company.

Bollens, E., De Proft E., \& Clarys, J. (1987). The accuracy and muscle monitoring in soccer kicking. In Jonsson, B. (Eds.), Biomechanics $X-A$. (pp. 283-288). Champaign Illinois: Human Kinetics.

Brophy, R. H., Backus, S. I., Pansy B. S., Lyman, S., \& Williams, R. J. (2007). Lower extremity muscle activation and alignment during the soccer instep and side- foot kicks. Journal of Orthopedic and Sports Physical Therapy, 37(5), 260- 268.

Cerrah, A. O., Onarici Gungor, E., Soylu A. R., Ertan, H., Lees, A., \& Bayrak, C. (2011). Muscular Activation patterns during the Soccer In- Step Kick. Isokinetic and Exercsice Science, 19(3), 181-190.

Clarys, J.P., Dufour, W., \& Pynaert, M. (1984). Electromyography of three soccer kick movements. Geneeskunde en Sport, 17, 17-21.

Commetti, G., Maffiuletti, N. A., Pousson, M., Chatard, J. C., \& Maffulli, N. (2001). Isokinetic strength and anaerobic power of elite, subelite and amateur French soccer players. International Journal of Sports Medicine, 22(1), 45-51.

De Proft, E., Clarys, J., \& Bollens, E. (1988). Muscle activity in the soccer kick. In Reilly, T., Lees, A., Davids K., \& Murphy, W.J. (Eds). Science and football (pp. 434-440). London: E \& FN Spon.

Domholdt, E. (2000). Physical therapy research. Principles and Applications: Philadelphia: WB Saunders.

Sørensen, H., Simonsen, E. B., Aagaard, H., Dyhre-Poulsen, P. \& Klausen, K. (1999). EMG activity of the iliopsoas muscle and leg kinetics during the soccer place kick. Scandinavian Journal Medicine Science and Sports, 9(4), 155-200.

Hermens, H. J., Freriks, B., Disselhorst-Klug, C., \& Rau, G. (2000). Development of recommendations for SEMG sensors and sensor placement procedures. Journal of Electromyography and Kinesiology, 10(5), 361-374.

Katis, A., \& Kellis, E. (2010). Three dimensional kinematics and ground reaction forces during the instep and outstep soccer kicks in pubertal players. Journal of Sports Sciences, 28(11), 1233-1241.

Kawamoto, R., Miyagi, O., Ohashi, J., \& Fukashiro, S. (2007). Kinetic comparison of a side-foot soccer kick between experienced and in experienced players. Sports Biomechanics, 6(2), 187-198.

Kellis, E., \& Baltzopoulos, V. (1998). Muscle activation differences between eccentric and concentric isokinetic exercise. Medicine Science in Sports and Exercise, 30(11), 16-23.

Kellis, E., Katis, A., \& Vrabas, I.S. (2006). Effects of an intermittent exercise fatigue protocol on biomechanics of soccer kick performance. Scandinavian Journal of Medicine and Science in Sports, 16(5), 334-344.

Kellis, E., \& Katis, A. (2007). Biomechanical characteristics and determinants of instep soccer kick. Journal of Sports Science and Medicine, 6(2), 154-165.

Lees, A., \& Nolan, L. (1998). The biomechanics of soccer: A Review. Journal of Sports Sciences, 16(3), 211-234.

Lees, A., \& Nolan, L. (2002). Three-dimensional kinematic analysis of the instep kick under speed and accuracy conditions. Science and football IV, 16-21.

Lees, A., Asai, T., Andersen, T. B., Nunome, H., \& Sterzing, T. (2010). The biomechanics of kicking in soccer: A review. Journal of sports sciences, 28(8), 805-817.

Levanon, J., \& Dapena, J. (1998). Comparison of the kinematics of the full-instep and pass kicks in soccer. Medicine and science in sports and exercise, 30(6), 917-927.

Masuda, K., Kikuhara, N., Takahashi, H., \& Yamanaka, K. (2003). The relationship between muscle crosssectional area and strength in various isokinetic movements among soccer players. Journal of sports sciences, 21(10), 851-858.

McCrudden, M., \& Reilly, T. (1993). A comparison of the punt and the drop-kick. Science and football II, 362-368.

Nunome, H., Asai, T., Ikegami, Y., \& Sakurai, S. (2002). Three-dimensional kinetic analysis of side-foot and instep soccer kicks. Medicine and science in sports and exercise, 34(12), 2028-2036.

Nunome, H., Lake, M., Georgakis, A., \& Stergioulas, L. K. (2006). Impact phase kinematics of instep kicking in soccer. Journal of Sports Sciences, 24(1), 11-22.

Scurr, J. C., Abbott, V., \& Ball, N. (2011). Quadriceps EMG muscle activation during accurate soccer instep kicking. Journal of sports sciences, 29(3), 247-251.

Shan, G., \& Westerhoff, P. (2005). Soccer: Full-body kinematic characteristics of the maximal instep soccer kick by male soccer players and parameters related to kick quality. Sports Biomechanics, 4(1), 59-72.

Sterzing, T., Lange, J. S., Wächtler, T., Müller, C., \& Milani, T. L. (2009). Velocity and accuracy as performance criteria for three different soccer kicking techniques. In ISBS-Conference Proceedings Archive (Vol. 1, No. $1)$. 